Background and Objectives Identification of biomarkers for early diagnosis and treatment response would be beneficial for patients with early rheumatoid arthritis (ERA) to prevent ongoing joint damage. MiRNAs have features of potential biomarkers and an altered expression of miRNAs was shown in established RA. Our aim was to analyse RA-associated miR-223 and miR-16 in sera from patients with ERA to find markers of early disease, clinical activity or predictors of disease outcome.

Materials and Methods Clinical characteristics were obtained in 34 patients with ERA at baseline and after 3 (M3) and 12 (M12) months therapy with DMARDs. Total RNA was isolated using phenol-chloroform extraction from whole sera obtained at baseline and M3. Peripheral blood mononuclear cells (PBMC) from healthy donors were treated with methotrexate (MTX, $25 \mathrm{ug} / \mathrm{ml}$ ) in vitro. Expression of miR-223 and miR-16 was analysed by TaqMan Realtime PCR.

Results Levels of miR-223 significantly decreased following therapy $(p=0.002)$. In treatment naïve patients with ERA, the expression of miR-223 positively correlated with baseline DAS28 $(p=0.031)$, change in DAS28 ( $\triangle \mathrm{DAS} 28)$ from baseline to $M 3$ $(p=0.014)$, baseline CRP ( $p=0.008)$ and count of peripheral leukocytes $(p=0.007)$. The change in expression of miR-223 in sera may be attributable to the change in the count of leukocytes between baseline and $\mathrm{M} 3$ concluded from the positive correlations between these variables $(p=0.025)$. In addition, the expression of miR-223 in PBMC was down regulated by $15 \%(p=0.001)$ after treatment with MTX

Levels of miR-16 significantly increased $(p=0.008)$ after 3 months of therapy and the increase in miR-16 was associated with the decrease in DAS28 from M3 to M12 ( $p=0.002)$.

Conclusions Our data support the potential of miR-223 to serve as a marker of disease activity in patients with treatment naïve ERA. Moreover, monitoring levels of miR-16 and miR-223 may become a useful tool to predict the disease outcome in patients with ERA.

Acknowledgement This work was supported by IMI BTCure, IAR, Masterswitch-PF7, Articulum, OPPA and MH CR project No. 023728.

\section{A7.4 ASSOCIATION OF GALECTIN SINGLE NUCLEOTIDE POLYMORPHISMS WITH AUTOIMMUNE DISEASES}

doi:10.1136/annrheumdis-2013-203221.4

1.2Z Pál, ${ }^{3 P}$ Antal ' ${ }^{1}$ SK Srivastava, ${ }^{4} \mathrm{~J}$ Gál, ${ }^{5} \mathrm{~S}$ André, ${ }^{6} \mathrm{E}$ Gordeeva, ${ }^{1} \mathrm{G}$ Nagy, ${ }^{5} \mathrm{H}$ Kaltner, ${ }^{6} \mathrm{NV}$ Bovin, ${ }^{2} \mathrm{MJ}$ Molnár, ${ }^{1} \mathrm{~A}$ Falus, ${ }^{5} \mathrm{HJ}$ Gabius, ${ }^{1} E \mathrm{E}$ Buzás. 'Department of Genetics, Cell- and Immunobiology, Semmelweis University, Budapest, Hungary; ${ }^{2}$ Department of Neurology, Semmelweis University, Budapest; ${ }^{3}$ University of Technology and Economics, Budapest, Hungary; " Department of Rheumatology, County Hospital, Kecskemét, Hungarys, 5/nstitute of Physiological Chemistry, Faculty of Veterinary Medicine, Ludwig-MaximiliansUniversity Munich, Munich, Germany; ${ }^{6}$ Shemyakin and Ovchinnikov Institute of Bioorganic Chemistry RAS, Moscow, Russia

Background and Objectives Galectins are potent immune regulators. Surprisingly, genetic association of galectin genes with autoimmune diseases have not yet been studied. A polymorphism in the coding region of the galectin-8 gene (Rs2737713; F19Y) and a novel galectin- 1 and interleukin 2 receptor $\beta$ haplotype were investigated for association with rheumatoid arthritis and myasthenia gravis.

Materials and Methods A case-control analysis and a related quantitative trait-association study were performed to investigate the association of the galectin 8 gene polymorphism in patients (myasthenia gravis 149, rheumatoid arthritis 214 and 134 as primary and repetitive cohorts, respectively) and 365 ethnically matched (Caucasian) healthy controls. Distribution was also investigated in patients grouped according to their antibody status and age at disease onset. Comparative testing for lectin activity was carried out in ELISA/ELLA-based binding tests with both wild-type and F19Y mutant galectin-8 from peripheral blood mononuclear cell lysates of healthy individuals with different genotypes as well as with recombinant wild-type and F19Y mutant galectin-8 proteins. Furthermore, we evaluated the association of regulatory region polymorphisms of the LGALS1 (Rs4820293, Rs4820294) and IL2R $\beta$ (Rs743777, Rs228941) genes in 146 Caucasian myasthenia gravis patients compared to 291 ethnically matched controls.

Results We found a strong association of the F19Y galectin 8 gene polymorphism with rheumatoid arthritis, and a mild one with myasthenia gravis. Moreover, the polymorphism also correlated with age at disease onset in the case of rheumatoid arthritis. The F19Y substitution did not appear to affect carbohydrate binding in solid-phase assays markedly. Also, a significant difference was found in the distribution of the Rs4820293/Rs743777 polymorphism haplotypes $(p<0.01)$ in patients with myasthenia gravis and controls but not in rheumatoid arthritis. The Rs4820293 polymorphism of LGALS1, previously not described to be associated with any disease, did not affect LGALS1 expression in peripheral mononuclear cells and skeletal muscle.

Conclusions This is the first study of an association between a galectin-based polymorphisms leading to a mutant protein and autoimmune diseases, with evidence for antagonistic pleiotropy.

\section{A7.5 COMBINED ANALYSIS OF EPIGENETIC AND TRANSCRIPTIONAL PROFILES IN DIFFERENT IMMUUNE CELLS IDENTIFIES HOT SPOTS OF GENE REGULATION BY DNA METHYLATION}

doi:10.1136/annrheumdis-2013-203221.5

'Marc Bonin, ${ }^{2}$ Stephan Flemming, ${ }^{2}$ Stefan Günther, ${ }^{3}$ Andreas Grützkau, ${ }^{1}$ Thomas Häupl. 'Department of Rheumatology and Clinical Immunology, Charité University Hospital, Berlin; ${ }^{2}$ Institute of Pharmaceutical Sciences, University of Freiburg; ${ }^{3}$ German Arthritis Research Center, Berlin

Background and Objectives Methylation of DNA may contribute to the regulation of gene expression. Chip technology enables to analyse for methylation of $\mathrm{CpG}$ sites but requires a pre-selection of potential hot spots. Such a selection of sites is represented on the HumanMethylation 450 array (Illumina). In order to test these CpG sites for possible functional effects, gene expression and DNA methylation were investigated between different immune cell types.

Materials and Methods Cells from 4 healthy donors were sorted by FACS technology for naive and memory T-cells (CD4m, CD4n, CD8m, Cd8n), B-cells (CD19m, CD19n), NK-cells (CD56), monocytes (CD14), and granulocytes (CD15). Genome-wide DNA methylation was assessed using the Illumina HumanMethylation 450 BeadChip platform. Analysis of data was performed using GenomeStudio (Illumina). Gene expression data were collected from Affymetrix HG-U133P transcriptomes analysed in the BioRetis database. Mapping of $\mathrm{CpG}$ sites with genes was performed using the ensemble genome assembly GRCh37 genomic location map.

Results The number of differentially expressed genes or methylated $\mathrm{CpG}$ sites were highest between very different cell types like CD14 monocytes and CD4 T-cells (4624 genes; 19261 sites) and lower between naive and memory cells of the same lymphocyte subtype (CD4: 638 genes; 9412 sites). There was a tendency towards more methylation in naive (CD4n: 5433 sites $\approx 2694$ genes) compared to memory cells (CD4m: 3979 sites $\approx 2258$ genes). Overlap of differential expression with corresponding changes in methylation was found in only 629 (279) of 1951 increased (2673 decreased) expressed genes for CD14 versus CD4 comparison and 57 (53) of 332 (306) genes for CD4m versus CD4n cells. Of all CpG sites annotated to these identified genes, only about $10 \%$ were concordant with expression. These CpG site were within or immediately upstream of the annotated start of the gene with a maximum 